\title{
Editorial: Stability: A Current Overview
}

\author{
F. Guarracino ${ }^{1 *}$ and A. Hutt ${ }^{2}$ \\ ${ }^{1}$ University of Naples "Federico II", Naples, Italy, ${ }^{2}$ Inria Nancy - Grand-Est Research Centre, Villers-lès-Nancy, France
}

Keywords: stability, buckling, dynamics, elasticity, plasticity, data assimilation

Editorial on the Research Topic

\section{Stability: A Current Overview}

The stability of equilibrium paths and of the evolution of dynamical systems is a classic field which has broad implications in virtually any field of science, from physics to human sciences, and ubiquitous applications from engineering to economics. Stability also plays an important role in the theory of differentiable mappings and, more generally, in the classification of geometrical objects.

As stated some thirty years ago by H. Leipholz in his treatise on Stability Theory [1], there is no absolute definition of stability. In fact, this concept has continuously evolved over the course of history and has been adjusted to the special requirements of particular problems in different fields. In this respect, various stability definitions have also contributed to the division. Restricting attention to the motion of planets, Lagrange, for example, stated that the trajectory of a planet can be defined as stable if its major axis remains bounded in spite of perturbations, while Poisson stated that the trajectory of a planet is stable when it repeatedly passes arbitrarily close to every point of its motion. Following these rather different definitions, the stability concept of Lyapunov has since found plentiful acceptance, but it has nevertheless remained not all-inclusive.

Also, from a methodological standpoint, investigations in stability have seen a progressive shift from analytical to differential-topological methods. In fact, as far back as the late 19th century

OPEN ACCESS

Edited and reviewed by: Fabio Lamantia, University of Calabria, Italy

${ }^{*}$ Correspondence:

F. Guarracino fguarrac@unina.it

Specialty section:

This article was submitted to

Dynamical Systems,

a section of the journal Applied

Mathematics and Statistics

Received: 25 September 2020

Accepted: 02 October 2020

Published: 12 November 2020

Citation:

Guarracino F and Hutt A (2020)

Editorial: Stability: A Current Overview.

Front. Appl. Math. Stat. 6:610153.

doi: 10.3389/fams.2020.610153
Poincarè proposed to pursue qualitative information by means of geometric methods. In this manner, many researchers were successively able to show the existence of periodic solutions in the three-body problem by applying differential-topological theorems to the phase portraits in addition to analytical methods. However, no analytical description of these orbits has been given, even if in some cases the orbits have been plotted numerically by computers. As such, numerical procedures have gained a significant role in stability investigations.

In mathematics, following the formulation of the problem of structural stability by Andronov and Pontriagin in 1937 [2], which is probably the most comprehensive of the many different definitions of stability, it is generally accepted that stability theory investigates the variation of the phase portrait of a dynamical system under small variations of the system itself by means of diffeomorphisms, or the variation of the global properties of a differentiable map between two manifolds, by means of diffeomorphisms-or homeomorphisms-on the manifolds.

Noticeably, even the stability of structures, a problem which was first investigated by Euler in the 18th century, has been, and to a certain extent remains, an old conundrum proven by the consistent disagreement between theoretical and experimental results. Over the past century, this required the introduction of various knockdown factors based on deterministic or probabilistic approaches.

The same applies to the stability of ergodic systems, whose stochastic treatment of initial parameters has often proven inaccurate in predicting their evolution. All these systems are affected by small perturbations and it has been believed that an approach based on appropriate statistical information could lead to a rational prediction of their behavior and evolution. 
The four articles that contribute to this Research Topic cover many of the previously illustrated fundamentals aspects of stability.

The first contribution by Champney et al. [Champney et al] places the emphasis on elastic structures that lose stability subcritically and shows that, despite practical stability assessments that may require stochastic approaches and imperfection maps, the buckling process and ultimate postbuckled state can nevertheless be well-described by the perfect problem. The state of the art is presented on analytical, numerical, and experimental methods and it is discussed how unstable states can be accessed by careful control of constraints and margins assessed by shock sensitivity experiments. The fact that subcritical instabilities can be robust, not being undone by reversal of the loading path, is shown to open up potential for technological exploitation.

In the second contribution [Mascolo], I. Mascolo focuses on the dynamic stability of elastic structures. The basic features of both linear and non-linear systems are discussed together with the effects of linearization on their phase portraits in the case of the so-called "degenerate bifurcations". In addition to a review of the main theoretical issues in the relevant literature, the paper illustrates the main methods (analytical, semi-analytical, and numerical) for dynamic stability analysis, highlighting their drawbacks and advantages. A focus on the imperfection sensitivity of dynamic stability problems is provided by means of the well-known Ziegler's paradox, concerning the effects of small damping. The problems of experimental testing in the realm of dynamic instability, especially with respect to nonconservative systems, is also discussed. Finally, the author shows how the investigation of chaotic and highly non-linear behavior in advanced applications can offer new opportunities in engineering design.

The third contribution by R. Shamass [Shamass] deals with stability problems in the framework of non-elastic deformations and illustrates some recent results with respect to the "plastic

\section{REFERENCES}

1. Leipholz H. Stability theory. 2nd ed. Stuttgard: Wiley (1987)

2. Andronov AA, Pontryagin LS. Systemes grossieres. Dokl Akad Nauk SSSR (1937) 14, 247-50.

Conflict of Interest: The authors declare that the research was conducted in the absence of any commercial or financial relationships that could be construed as a potential conflict of interest. buckling paradox", i.e. the circumstance that, notwithstanding that there is a general agreement that the deformation theory of plasticity lacks physical rigor in comparison to the flow theory, the use of the deformation theory has been repeatedly reported to predict buckling loads that are in better agreement with the experimental results. Until recently, this paradox was considered unsolved. It is shown that a formulation capable of offering a correct insight into the mechanics of the problem is able to attain a good agreement with the experimental results and provide reliable predictions for a large number of cases.

In the fourth contribution [Hutt], A. Hutt does not focus on strictly mechanical problems and rather makes reference to data assimilation, a technique developed to combine experimental data and a dynamical model, whose solutions might not actually result in accurate predictions of the real system dynamics. The technique works well for local observations, but it may turn unsatisfactory if the provided data is nonlocal, that is if the observation is a scalar sum of system activity. An example is the radiation measured from a satellite, which represents an integral value of the radiation of the whole atmosphere. The paper considers one of the most powerful data assimilation techniques, the Localized Ensemble Transform Kalman Filter (LETKF), used in several applications in meteorology and seismology, shows how to choose parameters of the LETKF, and how to adapt them to the properties of the observation operator to render the LETKF stable.

Overall, the papers provide an updated and fairly comprehensive picture of the ongoing research in the field and of its promising perspectives.

\section{AUTHOR CONTRIBUTIONS}

FG has conceived the structure of the Editorial and $\mathrm{AH}$ has contributed to the writing.

Copyright (c) 2020 Guarracino and Hutt. This is an open-access article distributed under the terms of the Creative Commons Attribution License (CC BY). The use, distribution or reproduction in other forums is permitted, provided the original author(s) and the copyright owner(s) are credited and that the original publication in this journal is cited, in accordance with accepted academic practice. No use, distribution or reproduction is permitted which does not comply with these terms. 